\title{
Leucine-Rich Alpha-2-Glycoprotein 1 in Serum Is a Possible Biomarker to Predict Response to Preoperative Chemoradiotherapy for Esophageal Cancer
}

\author{
Madoka Nambu, ${ }^{a}$ Takeshi Masuda,,${ }^{a, b, c}$ Shingo Ito, ${ }^{a, b, c}$ Ken Kato, ${ }^{d}$ Takashi Kojima,${ }^{e}$ \\ Hiroyuki Daiko, ${ }^{f \dagger}$ Yoshinori Ito, ${ }^{g}$ Kazufumi Honda, ${ }^{c, h}$ and Sumio Ohtsuki*a,b,c \\ ${ }^{a}$ Department of Pharmaceutical Microbiology, School of Pharmacy, Kumamoto University; 5-1 Oe-honmachi, \\ Chuo-ku, Kumamoto 862-0973, Japan: ${ }^{b}$ Faculty of Life Sciences, Kumamoto University; 5-1 Oe-honmachi, Chuo- \\ ku, Kumamoto 862-0973, Japan: ${ }^{\circ}$ AMED-CREST, Japan Agency for Medical Research and Development; 1-7-1 \\ Otemachi, Chiyoda-ku, Tokyo 100-0004, Japan: ${ }^{d}$ Department of Gastrointestinal Medical Oncology, National \\ Cancer Center Hospital; 5-1-1 Tsukiji, Chuo-ku, Tokyo 104-0045, Japan: ${ }^{\circ}$ Department of Gastroenterology and \\ Gastrointestinal Oncology, National Cancer Center Hospital East; 6-5-1 Kashiwanoha, Kashiwa, Chiba 277-8577, \\ Japan: ${ }^{f}$ Division of Gastrointestinal Oncology, National Cancer Center Hospital; 5-1-1 Tsukiji, Chuo-ku, Tokyo \\ 104-0045, Japan: ${ }^{g}$ Department of Radiation Oncology, Showa University School of Medicine; 1-5-8 Hatanodai, \\ Shinagawa, Tokyo 142-8666, Japan: and ${ }^{h}$ Department of Biomarkers for Early Detection of Cancer, National Cancer \\ Center Research Institute; 5-1-1 Tsukiji, Chuo-ku, Tokyo 104-0045, Japan.
}

Received May 13, 2019; accepted July 6, 2019

The purpose of the present study was to identify a biomarker that can predict the response to preoperative chemoradiotherapy (PCRT) in esophageal cancer patients. Twenty-five serum samples collected from patents with esophageal cancer before PCRT (responder $=13$, non-responder $=12$ ) were analyzed by quantitative proteomics, and 248 proteins were identified. Among them, the serum levels of leucine-rich alpha-2-glycoprotein 1 (LRG1) were significantly different $(p<0.01)$ and well discriminated (area under the curve $(A U C)$ of the receiver operating characteristic (ROC) curve $>0.8$ ) between responder and nonresponder groups. The combination of LRG1 with C-reactive protein (CRP) and soluble interleukin-6 receptor (sIL-6R), which were previously reported as biomarkers predicting PCRT response, further improved the predictive performance, providing an $A U C$ of greater than 0.9. The present results suggest that LRG1 and its combination with CRP and sIL-6R are promising biomarker candidates to predict response to PCRT in esophageal cancer patients. teomics

Key words biomarker; esophageal cancer; chemoradiotherapy; leucine-rich alpha-2-glycoprotein 1; pro-

\section{INTRODUCTION}

Esophageal cancer is the 6th leading cause of cancer-related death in the world, and is one of the most deadly cancers. ${ }^{1)}$ Esophageal cancer has a significantly higher incidence in males, and it was reported that smoking and drinking are risk factors. $^{2,3)}$ According to a joint survey of survival rates of Japanese Association of Clinical Cancer Centers (April 2018), the 5-year survival rate after diagnosis of esophageal cancer is $43.3 \%$.

Although preoperative chemoradiotherapy (PCRT) before surgical resection of resectable esophageal cancer has been reported to improve prognosis, ${ }^{4)}$ PCRT does not improve the survival of all patients. ${ }^{5}$ Moreover, chemoradiotherapy has toxic side effects, and treatment takes more than 1 month. ${ }^{6}$ In addition, it is difficult to apply chemotherapy of adequate strength to control micrometastases from primary sites, due to the toxicity. Therefore, to avoid ineffective treatment with potentially severe adverse effects, it is very important to identify patients in whom PCRT is, or is not, likely to be effective. Patients for whom PCRT is predicted not to be effective should

${ }^{\dagger}$ Present address: Division of Esophageal Surgery, National Cancer Center Hospital; 5-1-1 Tsukiji, Chuo-ku, Tokyo 104-0045, Japan. be given preoperative chemotherapy as standard therapy. Therefore, we require a biomarker that can predict whether or not PCRT will be effective prior to the treatment.

It has been reported that tissue expression of programmed cell death 4 protein, nuclear factor $\kappa \mathrm{B}$, or inhibitor of kappaB $(\mathrm{I} \kappa \mathrm{B})$ kinase epsilon in esophageal cancer is associated with clinical outcomes. ${ }^{7-9)}$ On the other hand, as regards blood biomarkers, it is reported that elevation of preoperative serum Creactive protein (CRP) levels is related to poor prognosis and poor PCRT response in patients with resectable esophageal cancer. ${ }^{10-12)}$ In a previous study with multiplex immunobeadsbased assay of 84 cytokines, we identified soluble interleukin-6 receptor (sIL-6R) as a serum biomarker for the response of esophageal cancer to neoadjuvant chemoradiotherapy. $\left.{ }^{6}\right)$ However, the abilities of CRP and sIL-6R to discriminate PCRT responders from non-responders were limited. ${ }^{6)}$ Thus, a better biomarker is needed to predict the response to PCRT.

Proteome analysis of human serum and plasma is a standard strategy to identify blood biomarkers of various diseases. Furthermore, improvements in proteomics methodologies have enabled deep analysis of the blood proteome. Therefore, the purpose of the present study was to identify a novel blood biomarker for predicting patients' response to PCRT by means of quantitative proteomics. 


\section{MATERIALS AND METHODS}

Serum Samples Serum samples were collected from 26 stage II-III (excluding T4) patients, who were identified as the prospective PCRT cohort in our previous report, ${ }^{6)}$ and enrolled in the accompanying phase II clinical trial of neoadjuvant PCRT at the National Cancer Center Hospital and the National Cancer Center Hospital East during 2010 and 2011. ${ }^{13)}$ One serum sample was not available for the present study due to disqualification of the patient from the trial. The 25 patients whose serum samples were used in the present study are listed in Table 1. Samples collected prior to treatment were stored frozen until analysis. Patients received two courses of protracted 5-fluorouracil infusion $\left(1000 \mathrm{mg} / \mathrm{m}^{2} / \mathrm{d}\right)$ on days 1-4 and days 29-32, and 2-h infusions of cisplatin on days 1 and $29\left(75 \mathrm{mg} / \mathrm{m}^{2}\right)$, with hydration and antiemetic coverage, together with concurrent irradiation $(1.8 \mathrm{~Gy} / \mathrm{d})$. Surgical resection was done 6-8 weeks after the end of PCRT. Histological responses were classified into Grades 0-3 (G0, G1, G2, and G3) according to the Japanese Classification of Esophageal Cancer (9th ed.). Responders are the patients who responded to PCRT (G3, $n=12$ ), and non-responders are those who did not (G1 and G2, $n=13)$. Serum levels of CRP and sIL-6R were determined in our previous report. ${ }^{6}{ }^{6}$ This study was approved by the Ethics Committees of Kumamoto University (No. 1144) and National Cancer Center (No. 2015-041), and was conducted in accordance with the Declaration of Helsinki. Written informed consent was obtained from all participants.

Quantitative Proteomic Analysis The details of the proteomics analysis are given in the supplementary materials. Briefly, the serum samples were digested with lysyl endopeptidase and trypsin. To identify biomarker candidate peptides, the peptide-containing solutions were subjected to nanoLCelectrospray ionization (ESI)-MS/MS, and data was acquired in data-dependent and data-independent acquisition modes. To validate the LRG peptides, the peptides were quantified by microLC-ESI-MS/MS after spiking stable isotope-labeled peptides as internal standards.
Statistical Analysis The normality and equality of variance of the data were determined by means of the Shapiro-Wilk test and $F$-test, respectively, using Origin2019 (OriginLab, Northampton, MA, U.S.A.). Statistical power was also determined using Origin2019 (OriginLab). The statistical significance of differences was determined by employing Student's $t$-test using Microsoft Excel (Microsoft, Seattle, WA, U.S.A.), Fisher's exact $t$-test and the chi-square test using GraphPad Prism 7.04 (GraphPad, San Diego, CA, U.S.A.), and Welch's $t$-test and the Mann-Whitney $U$-test using Origin2019 (OriginLab), as appropriate. The area under the curve $(A U C)$ of receiver operating characteristic (ROC) curves was analyzed by means of GraphPad Prism 7.04 (GraphPad). Multivariate logistic regression analysis was performed using R-3.3.2 for Windows (Free Software Foundation, Boston, MA, U.S.A.).

\section{RESULTS}

Table 1 shows information on sex, age, clinical stage of esophageal cancer, and prognosis of patients. None of these factors showed a significant difference between responder and non-responder groups. In serum samples collected before initiation of any treatment, there was a significant difference in CRP levels $(p=0.0363)$ and no significant difference in sIL6R levels $(p=0.117)$ between the groups.

By means of quantitative proteomic analysis, 248 proteins were identified from 3657 tryptic peptides based on quantification of all 25 samples. Among the identified peptides, 9 peptides were selected as biomarker candidates to discriminate patients who responded to PCRT (G3, $n=12)$ from those who did not (G1 and G2, $n=13$ ) according to the criteria shown in the footnote of Table 2. Among these candidates, 6 peptides were trypsin-digested peptides of leucine-rich alpha-2-glycoprotein 1 (LRG1). Unique tryptic peptides derived by digestion from the same protein are expected to give essentially the same $A U C$ values of the ROC curve and fold differences as those of the parental protein. Indeed, the 6 digested peptides

Table 1. Characteristics of Subjects

\begin{tabular}{|c|c|c|c|}
\hline \multirow{2}{*}{ Variable } & Non-responder & Responder & \multirow{2}{*}{$p$-Value } \\
\hline & $\mathrm{G} 1$ and $\mathrm{G} 2(n=12)$ & $\mathrm{G} 3(n=13)$ & \\
\hline \multirow[t]{2}{*}{ Age } & $62.1 \pm 6.2$ & $62.7 \pm 7.5$ & n.s. $(0.331)$ \\
\hline & $63(60-65)$ & $64(60-68)$ & \\
\hline \multicolumn{4}{|l|}{ Sex } \\
\hline Male & 10 & 13 & n.s. $(0.220)$ \\
\hline Female & 2 & 0 & \\
\hline \multicolumn{4}{|l|}{ Clinical stage } \\
\hline IIA & 1 & 0 & n.s. $(0.241)$ \\
\hline IIB & 3 & 7 & \\
\hline III & 8 & 6 & \\
\hline \multicolumn{4}{|c|}{ Protein level in serum } \\
\hline \multirow[t]{2}{*}{ CRP (mg/mL) } & $4.90 \pm 7.4$ & $2.86 \pm 4.6$ & 0.0363 \\
\hline & $0.744(0.610-9.80)$ & $0.477(0.297-5.53)$ & \\
\hline \multirow[t]{2}{*}{ sIL-6R (ng/mL) } & $25.7 \pm 6.6$ & $21.7 \pm 5.9$ & n.s. $(0.117)$ \\
\hline & $26.0(20.2-28.0)$ & $21.3(17.1-26.1)$ & \\
\hline
\end{tabular}

Age, CRP and sIL-6R are shown as the mean \pm standard deviation (S.D.) in the upper line and the median (Q1-Q3) in the lower line. The significance of differences was determined by employing the Mann-Whitney $U$-test (age, CRP), Student's $t$-test (sIL-6R), Fisher's exact $t$-test (sex), or the chi-square test (clinical stage). n.s., not significantly different $(p>0.05)$ 
Table 2. Amino Acid Sequences of Identified Peptides for Marker Candidate Proteins

\begin{tabular}{|c|c|c|c|c|}
\hline Protein & Peptide & $p$-Value ${ }^{a)}$ & Fold $^{b)}$ & $A U C^{c)}$ \\
\hline Alpha-1-acid glycoprotein 1 & GGQEHFAHLLILR & 0.00249 & 0.427 & 0.827 \\
\hline Haptoglobin & YSQVDIGLIK & 0.00679 & 0.571 & 0.814 \\
\hline Ig mu chain $\mathrm{C}$ region & LEWLGR & 0.00307 & 0.288 & 0.878 \\
\hline Leucine-rich alpha-2-glycoprotein & GQTLLAVAK & 0.00110 & 0.591 & 0.885 \\
\hline Leucine-rich alpha-2-glycoprotein & GPLQLER & 0.00146 & 0.641 & 0.859 \\
\hline Leucine-rich alpha-2-glycoprotein & DLLLPQPDLR & 0.00329 & 0.606 & 0.853 \\
\hline Leucine-rich alpha-2-glycoprotein & VAAGAFQGLR & 0.00456 & 0.621 & 0.846 \\
\hline Leucine-rich alpha-2-glycoprotein & ENQLEVLEVSWLHGLK & 0.00481 & 0.595 & 0.865 \\
\hline Leucine-rich alpha-2-glycoprotein & TLDLGENQLETLPPDLLR & 0.00768 & 0.637 & 0.821 \\
\hline
\end{tabular}

The peptides discriminating patients who responded to PCRT (G3) from those who did not (G1 and G2) were selected as biomarker candidates according to the following criteria: a significant difference by Student's $t$-test $(p<0.01)$, level in responder group greater than 1.5 -fold or smaller than 0.667 -fold compared to non-responders, and more than 0.8 AUC of the ROC curve. $a$ ) $p$-Values of Student's $t$-test between responder and non-responder groups. $b$ ) Fold difference between the mean values of responder group and non-responder group. c) $A U C$ of the ROC discriminating between responder group and non-responder group.

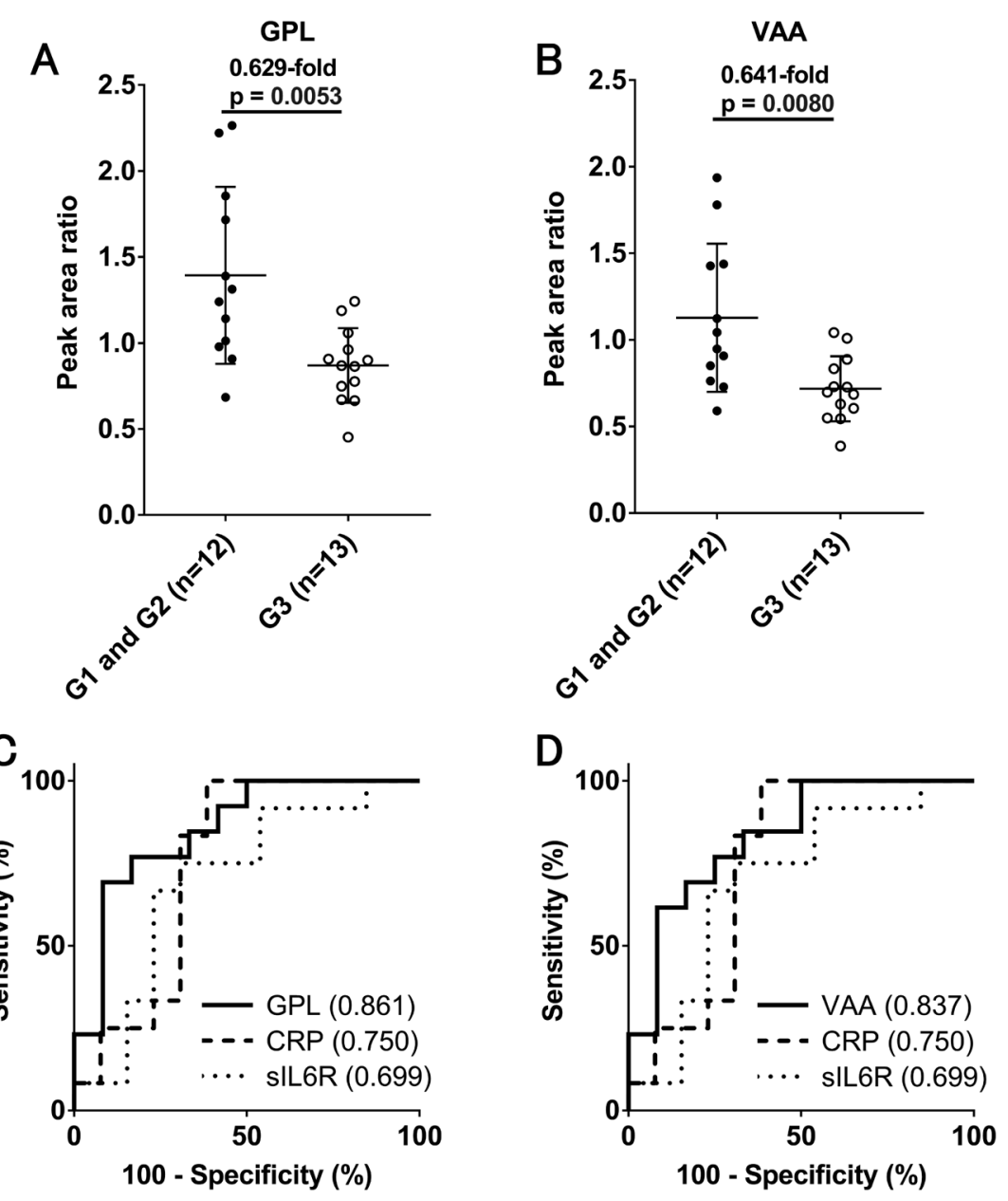

Fig. 1. Dot Plots and ROC Curves for Two Selected LRG1-Peptides

(A, B) Dot plots showing the differences of LRG1 peptide levels between PCRT non-responder (G1 and G2, $n=12)$ and responder (G3, $n=13)$ groups. Each symbol represents the protein level in an individual sample, and lines represent the mean and S.D. The fold value represents the mean ratio of responder group to non-responder group. Statistical significance was determined with Welch's $t$-test. (C, D) ROC curves of LRG1-peptides compared with those of CRP and sIL-6R. Values in parenthesis indicate the $A U C$ of the ROC curves.

from LRG1 (LRG1-peptides) displayed similar values of fold change and $A U C$, supporting the idea that LRG1 protein is a marker protein candidate.

We selected two LRG1-peptides, VAAGAFQGLR (VAApeptide) and GPLQLER (GPL-peptide), as representative peptides for validation by targeted proteomics based on high detection sensitivity and clear chromatographic peaks. These two LRG1-peptides were measured in all serum samples after spiking stable internal standard peptides having identical amino acid sequences. As shown in Figs. 1A and B, the levels of these LRG1-peptides in the responder group (G3) were significantly lower by 0.629 - and 0.641 -fold than those in the nonresponder group (G1 and G2). The statistical power to detect a difference at 0.05 was 0.897 for GPL-peptide and 0.855 for 

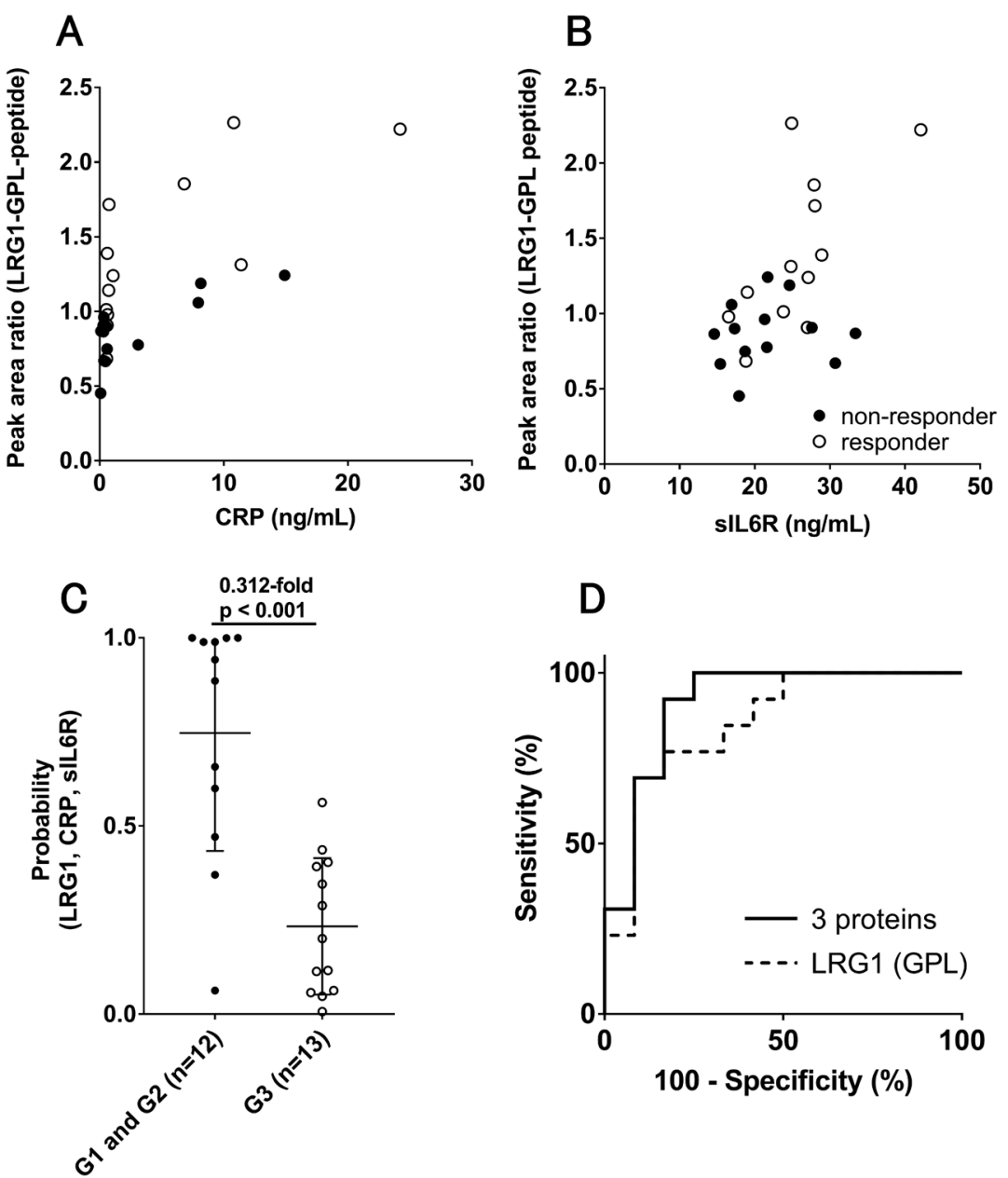

Fig. 2. Correlation of LRG1-GPL-Peptides to CRP and sIL-6R, and Regression Model with LRG1-GPL-Peptides, CRP and sIL-6R

Correlation of LRG1-GPL-peptides to CRP (A) and sIL-6R (B). Each symbol represents the level in an individual sample. Closed circles indicate non-responders, and open circles indicate responders. (C) Dot plots showing the differences of probability values calculated from the regression model with LRG1-GPL-peptides, CRP and sIL6R between PCRT responder (G3, $n=13)$ and non-responder (G1 and G2, $n=12)$ groups. The model is $p=1 /(1+\exp (9.31-10.5 *[\mathrm{GPL}]+0.0199 *[\mathrm{sIL} 6 \mathrm{R}]+0.360 *[\mathrm{CRP}]))$. Each dot represents the probability value in an individual sample, and lines represent the mean and S.D. The fold value represents the mean ratio of responder group to non-responder group. Statistical significance was determined with the Mann-Whitney $U$-test. (D) ROC curves of the regression model and LRG1-GPL-peptide.

VAA-peptide. The $A U C$ s of the ROC curves (0.861 and 0.837$)$ were greater than those of CRP (0.750) and sIL-6R (0.699) (Figs. 1C, D). Further, there was a high correlation between the levels of GPL- and VAA-peptides $(r=0.995, p<0.0001)$. Therefore, in the following analysis, we took the level of GPLpeptide as representing the level of LRG1 protein.

As shown in Figs. 2A and B, the correlation between LRG1-GPL-peptide and CRP $(r=0.685, p=0.0002)$ or sIL-6R $(r=0.553, p=0.0041)$ was significant, but weak. Therefore, we considered that combination of the 3 proteins as a multimarker panel might improve the performance to discriminate between responder and non-responder groups. ${ }^{14)}$ To test this idea, we performed multivariate logistic regression analysis using the levels of LRG1-GPL-peptide, CRP and sIL-6R as independent variables. Indeed, the probability values calculated from the regression model were significantly greater in the non-responder group (G1 and G2) than in the responder group (G3) $(p<0.001$, Fig. 2C), and the $A U C$ of the ROC curve was improved to 0.910 (Fig. 2D). This result suggests that the combination of the 3 proteins resulted in greater ability to discriminate between PCRT responders and non-responders.

\section{DISCUSSION}

The present study revealed that the serum level of LRG1 is significantly lower in pre-PCRT serum of patients who subsequently responded to PCRT, as compared with non-responders or poor responders (Fig. 1). LRG1 is a member of the leucine-rich repeat family in human serum, and was shown to promote angiogenesis by modulating endothelial transforming growth factor- $\beta$ (TGF- $\beta$ ) signaling. ${ }^{15)}$ It has been reported that high expression of angiogenic factors, such as thymidine phosphorylase and vascular endothelial cell growth factor, is associated with a low response to chemoradiotherapy in patients with esophageal squamous cell carcinoma. ${ }^{16)}$ Therefore, it is reasonable that high levels of LRG1 would be associated with a poor response to PCRT.

LRG1 is also related to inflammation. It is induced in the liver by the pro-inflammatory cytokine IL- $6 .{ }^{17)}$ Further, serum levels of LRG1 are elevated in patients with rheumatoid arthritis or ulcerative colitis. ${ }^{18,19)}$ As already mentioned, elevation of preoperative serum CRP and sIL-6R levels is related to poor PCRT response in patients with resectable esophageal cancer. ${ }^{6,10,11)}$ Serum CRP increases during inflammation, and CRP is reported to be regulated by IL- $6 .{ }^{20)}$ sIL-6R is a soluble form 
of IL-6 receptor that binds with IL-6, and activates cell signaling via interaction with membrane-bound gp130. ${ }^{21)}$ Since all three proteins are related to inflammation and IL-6 signaling, it appears that there is a close relation between inflammation and the response to PCRT. The radiation responsiveness of tumors is modulated by inflammation, and radiation induces various cytokines, including IL- $6 .{ }^{22)}$ In addition, the activation of the inflammatory response by lipopolysaccharide prior to radiation induced radioresistance in non-small cell lung cancer cells. ${ }^{23)}$

The expression of LRG1 is regulated by multiple cytokines, including IL-6. It is reported that the level of LRG1 mRNA was increased 2-fold in HepG2 cells in response to IL-6 and IL-1 $\beta$ co-treatment and 1.5 -fold in response to IL- 6 and tumor necrosis factor (TNF) $\alpha$ co-treatment compared with IL-6 single treatment. ${ }^{17)}$ Therefore, weak correlations between the levels of LRG1, sIL-6R and CRP might be explained by partial sharing of regulatory pathways and different relationships to PCRT response. Since the PCRT response would be affected by multiple factors due to tumor heterogeneity, the combined use of the 3 peptides as a multi-marker panel is expected to improve the predictive performance (Fig. 2).

In conclusion, by means of quantitative proteomic analysis, we have identified a novel blood biomarker, LRG1, for predicting the PCRT response of esophageal cancer patients. Further, the combination of LRG1, CRP and sIL-6R provided better discrimination of the responder group from the non-responder group than any single biomarker. To determine cut-off values for clinical diagnosis, it would be necessary to measure absolute values of LRG1, e.g., by enzyme-linked immunosorbent assay (ELISA). Further studies with a larger group of patients seem worthwhile to confirm the usefulness of the serum levels of LRG1, CRP and sIL-6R as predictors of PCRT response.

Acknowledgment This study was supported in part by AMED-CREST from Japan Agency for Medical Research and Development.

Conflict of Interest S.O. is a full professor at Kumamoto University and is also a director of Proteomedix Frontiers. This study was not supported by the company. The other authors declare no competing interests.

Supplementary Materials The online version of this article contains supplementary materials.

\section{REFERENCES}

1) Smyth EC, Lagergren J, Fitzgerald RC, Lordick F, Shah MA, Lagergren P, Cunningham D. Oesophageal cancer. Nat Rev Dis Primers, 3, 17048 (2017).

2) Cook MB, Chow WH, Devesa SS. Oesophageal cancer incidence in the United States by race, sex, and histologic type, 1977-2005. Br. J. Cancer, 101, 855-859 (2009).

3) Freedman ND, Abnet CC, Leitzmann MF, Mouw T, Subar AF, Hollenbeck AR, Schatzkin A. A prospective study of tobacco, alcohol, and the risk of esophageal and gastric cancer subtypes. Am. J. Epidemiol., 165, 1424-1433 (2007).

4) Crehange G, Bonnetain F, Peignaux K, Truc G, Blanchard N, Rat P, Chauffert B, Ghiringhelli F, Maingon P. Preoperative radiochemotherapy for resectable localised oesophageal cancer: a controversial strategy. Crit. Rev. Oncol. Hematol., 75, 235-242 (2010).
5) Osaka Y, Takagi Y, Tsuchida A, Hoshino S, Tachibana S, Shinohara M, Aoki T. Concurrent preoperative chemoradiotherapy for stage III or IV esophageal squamous carcinoma. Oncol. Rep., 12, 1121-1126 (2004).

6) Makuuchi Y, Honda K, Osaka Y, Kato K, Kojima T, Daiko H, Igaki $\mathrm{H}$, Ito $\mathrm{Y}$, Hoshino $\mathrm{S}$, Tachibana $\mathrm{S}$, Watanabe $\mathrm{T}$, Furuta $\mathrm{K}$, Sekine S, Umaki T, Watabe Y, Miura N, Ono M, Tsuchida A, Yamada T. Soluble interleukin-6 receptor is a serum biomarker for the response of esophageal carcinoma to neoadjuvant chemoradiotherapy. Cancer Sci., 104, 1045-1051 (2013).

7) Fassan M, Cagol M, Pennelli G, Rizzetto C, Giacomelli L, Battaglia G, Zaninotto G, Ancona E, Ruol A, Rugge M. Programmed cell death 4 protein in esophageal cancer. Oncol. Rep., 24, 135-139 (2010).

8) Izzo JG, Malhotra U, Wu TT, Luthra R, Correa AM, Swisher SG, Hofstetter W, Chao KS, Hung MC, Ajani JA. Clinical biology of esophageal adenocarcinoma after surgery is influenced by nuclear factor-kappaB expression. Cancer Epidemiol. Biomarkers Prev., 16, 1200-1205 (2007).

9) Yang W, Qu Y, Tan B, Jia Y, Wang N, Hu P, Wang J. Prognostic significance of preoperative IKBKE expression in esophageal squamous cell carcinoma. Onco. Targets Ther., 11, 1305-1314 (2018).

10) Badakhshi H, Kaul D, Zhao KL. Association between the inflammatory biomarker, C-reactive protein, and the response to radiochemotherapy in patients with esophageal cancer. Mol. Clin. Oncol., 4, 643-647 (2016)

11) Crumley AB, McMillan DC, McKernan M, Going JJ, Shearer CJ, Stuart RC. An elevated C-reactive protein concentration, prior to surgery, predicts poor cancer-specific survival in patients undergoing resection for gastro-oesophageal cancer. Br. J. Cancer, 94, 1568-1571 (2006).

12) Fujiwara $H$, Suchi $K$, Okamura $S$, Okamura $H$, Umehara $S$, Todo M, Shiozaki A, Kubota T, Ichikawa D, Okamoto K, Ochiai T, Kokuba Y, Sonoyama T, Otsuji E. Elevated serum CRP levels after induction chemoradiotherapy reflect poor treatment response in association with IL-6 in serum and local tumor site in patients with advanced esophageal cancer. J. Surg. Oncol., 103, 62-68 (2011).

13) Hashimoto J, Kato K, Ito $Y$, Kojima $T$, Akimoto $T$, Daiko $H$, Hamamoto Y, Matsushita H, Katano S, Hara H, Tanaka Y, Saito Y, Nagashima K, Igaki H. Phase II feasibility study of preoperative concurrent chemoradiotherapy with cisplatin plus 5-fluorouracil and elective lymph node irradiation for clinical stage II/III esophageal squamous cell carcinoma. Int. J. Clin. Oncol., 24, 60-67 (2019).

14) Hirata $Y$, Kobayashi $T$, Nishiumi S, et al. Identification of highly sensitive biomarkers that can aid the early detection of pancreatic cancer using GC/MS/MS-based targeted metabolomics. Clin. Chim. Acta, 468, 98-104 (2017).

15) Wang X, Abraham S, McKenzie JAG, Jeffs N, Swire M, Tripathi VB, Luhmann UFO, Lange CAK, Zhai Z, Arthur HM, Bainbridge J, Moss SE, Greenwood J. LRG1 promotes angiogenesis by modulating endothelial TGF-beta signalling. Nature, 499, 306-311 (2013).

16) Shimada H, Hoshino T, Okazumi S, Matsubara H, Funami Y, Nabeya Y, Hayashi H, Takeda A, Shiratori T, Uno T, Ito H, Ochiai T. Expression of angiogenic factors predicts response to chemoradiotherapy and prognosis of oesophageal squamous cell carcinoma. $B r$. J. Cancer, 86, 552-557 (2002).

17) Shirai R, Hirano F, Ohkura N, Ikeda K, Inoue S. Up-regulation of the expression of leucine-rich alpha(2)-glycoprotein in hepatocytes by the mediators of acute-phase response. Biochem. Biophys. Res. Commun., 382, 776-779 (2009).

18) Ha YJ, Kang EJ, Lee SW, Lee SK, Park YB, Song JS, Choi ST. Usefulness of serum leucine-rich alpha-2 glycoprotein as a disease activity biomarker in patients with rheumatoid arthritis. J. Korean Med. Sci., 29, 1199-1204 (2014).

19) Shinzaki S, Matsuoka K, Iijima H, Mizuno S, Serada S, Fujimoto M, Arai N, Koyama N, Morii E, Watanabe M, Hibi T, Kanai T, 
Takehara T, Naka T. Leucine-rich alpha-2 glycoprotein is a serum biomarker of mucosal healing in ulcerative colitis. J. Crohn's Colitis, 11, 84-91 (2017).

20) Nishikawa T, Hagihara $K$, Serada $S$, Isobe T, Matsumura A, Song J, Tanaka T, Kawase I, Naka T, Yoshizaki K. Transcriptional complex formation of c-Fos, STAT3, and hepatocyte NF-1 alpha is essential for cytokine-driven C-reactive protein gene expression. J. Immunol., 180, 3492-3501 (2008)

21) Jones SA, Horiuchi S, Topley N, Yamamoto N, Fuller GM. The soluble interleukin 6 receptor: mechanisms of production and implications in disease. FASEB J., 15, 43-58 (2001).

22) Multhoff G, Radons J. Radiation, inflammation, and immune responses in cancer. Front Oncol., 2, 58 (2012).

23) Kim W, Youn H, Kang C, Youn B. Inflammation-induced radioresistance is mediated by ROS-dependent inactivation of protein phosphatase 1 in non-small cell lung cancer cells. Apoptosis, 20, 1242-1252 (2015). 